\title{
The Effect of 8 Weeks of Complex Training Methods on the Countermovement Jump Performance
}

\author{
Tereza Králová', Tereza Hammerová1 Marián Vanderka², Jan Cacek', Michal Bozděch', Václav Vrbas \\ Masaryk University Faculty of Sports Studies Brno Czech Republic', \\ Comenius University in Bratislava Faculty of Physical Education and Sports Bratislava Slovak Republic ${ }^{2}$
}

\begin{abstract}
PURPOSE: The purpose of this study was to find out whether a more specific stimulus, such as training which stimulates the production of power above $90 \%$ of $P_{\max }\left(P_{\max }=\right.$ maximal average concentric power output), is effective in enhancing the countermovement jump (CMJ) performance using the method of complex pairs or separate execution of the exercises in the complex pair (separate complex pair).

METHODS: Thirty male students of the Faculty of Sports Studies were divided into 2 experimental (EX1, EX2) and 1 control group (CNTR). The experimental groups trained for 8 weeks using the complex training (CT) method twice a week with the same amount of repetitions for each exercise. The complex pair consisted of half-squat jumps with the intensity of over $90 \%$ of $P_{\text {max }}$ and plyometric depth-jumps. EX1 trained using the complex pair method with the intracomplex rest interval of up to 15 seconds. EX2 trained all sets of the half-squat jumps first and then all sets of the plyometric exercise. We used non-parametric statistics and linear regression analysis to evaluate the effect on increasing the CMJ performance after the intervention program.

RESULTS: There were no significant differences between the pre-test and post-test in any of the experimental groups $(p>0.05)$, although there were significant differences between the weeks in EX1 and EX2 $(p<0.05)$. The CMJ performance increased insignificantly $(p>0.05)$ each week by $0.14 \mathrm{~cm}$ in EX1 and by $0.07 \mathrm{~cm}$ in EX2.

CONCLUSION: These results did not clearly show the application of the CT methods to be useful in enhancing the CMJ. In practice, we recommend using complex pairs to save training time and increase training intensity. However, an additional longer-term intervention experiment with a bigger sample size and groups randomized only by the CMJ parameter (not by $P_{\max }$ and CMJ performance together) is needed.
\end{abstract}

Keywords: power output; Pmax; PAP; half-squat jumps; depth jumps, complex pairs

\section{INTRODUCTION}

Strength and power training is widely used by recreational and elite athletes in their training. Strength training and one of its forms, resistance training, is generally known as a method to promote many benefits such as improving physical strength and body posture (Molina-Garcia, Plaza-Florido, Mora-Gonzalez, Torres-Lopez, Vanrenterghem, \& Ortega, 2020), improving body composition (Zeng, Peng, Zhao, \& Chen, 2020). Furthermore, resistance training helps against insulin resistance (Niemann, Tucker, Bailey, \& Davidson, 2020) and also brings positive psychological impact on future lifestyle habits and thus reduces the risk of chronic diseases (Faigenbaum, Kraemer, Blimkie, Jeffreys, Micheli, Nitka, \& Rowland, 2009; Rowland, 2007). In the field of elite sports training, coaches and scientists work together to develop new training methods to improve and support their athletes to achieve better results effectively in a short time using resistance training with other forms of strength and power training. 
One of the commonly used effective methods is the shock method, also known as the plyometric method (Ozen, Atar, \& Koc, 2020). The plyometric exercises include jumping, throwing, bounding. Resistance training and plyometric training have been incorporated into weekly programs separately for several years. However, with the development of postactivation potentiation (PAP) and thus muscle toning enhancement which proved to be a good method for preparing the muscles for more power production (Robbins, 2005), the coaches put resistance and plyometric exercises together. One of the definitions of PAP is an increase in muscle twitch and lowfrequency tetanic force after a contractile activity (Sale, 2002). The current studies mostly focus on an immediate effect of PAP, and to a very limited extent on possible chronic adaptation to PAP. When including more sets of a heavy resistance exercise together with a plyometric exercise, it is possible to talk about complex training that uses increased muscle activation as a tool to increase the training intensity of the exercise. With its regular inclusion in the training program, we can focus on chronic adaptation. Doing a heavy resistance exercise before a plyometric exercise which is biomechanically similar is called the complex training method (Hodgson, Docherty, \& Robbins, 2005). Heavy resistance exercises associated with the application of complex training are in most recommendations defined as a load of at least over $80 \%$ of $1 \mathrm{RM}$ (repetition maximum) (Docherty, Robbins, \& Hodgson, 2004; Ebben, 2002). There are many recommendations and options for applying the complex training method. From the basic recommendation by Bompa (1983) to become a more versatile athlete to Chu (1998) and his recommendations to do heavy resistance exercises (like squats) and plyometrics (like a triple jump) in the same training session to today's view of the complex training.

Today's view is divided into two main approaches to and interpretations of complex pairs. The first one is doing heavy resistance exercises (all the sets) and then doing all the sets of the plyometric ones while maintaining biomechanical similarity of the exercises. The second interpretation, partly similar to the methodological approach of contrast training, is to perform the first set of a heavy resistance exercise and then immediately, or after a rest interval, do one set of a plyometric exercise while maintaining biomechanical similarity of the exercises as well. However, every recommendation included the load of the first heavy load exercise based only on the $\%$ of the $1 \mathrm{RM}$ value or RM value in repetitions. We did not find a study that would include $\%$ of power production and heavy power output exercises instead of heavy load (above $80 \%$ of $1 \mathrm{RM}$ ) exercises when investigating the effect of complex training. This is the reason why we tried to find out whether a more specific stimulus, training which stimulates the production of power above $90 \%$ of $\mathrm{P}_{\max }\left(\mathrm{P}_{\max }=\right.$ maximal average concentric power output), will be effective in enhancing the countermovement jump (CMJ) performance using the method of complex pairs or separate execution of the exercises in the complex pair (separate complex pair).

\section{METHODS}

\section{Experimental Approach to the Problem}

This study was designed to investigate the effect of an 8-week training program using complex pairs on the CMJ performance each week. Two different approaches to the complex training methods were used. One method implemented complex pairs (with the interval between exercises of up to $15 \mathrm{~s}$ ) and the second method consisted of performing all the sets of power-strength exercises first and then all the sets of plyometric exercises in the same training session (separate pair exercises). We also focused on bringing the conditions as close to real training as possible. Therefore, the load was regulated precisely, so that the athletes always trained above the value of 
$90 \%$ of their current $\mathrm{P}_{\max }$. This means that the load was tested at the beginning of the first workout of the week during the first set of half-squats jumps.

The 8-week experimental study consisted of 16 training sessions. The participants trained twice per week (minimum of 48 hours apart) for the duration of this study. The complex pair group (EX1) completed 8 complex pairs per session. One complex pair consisted of 4 repetitions of half-squat jumps with a load corresponding to a minimum of $90 \%$ of $\mathrm{P}_{\max }$ measured during the pre-test, immediately followed (within up to $15 \mathrm{~s}$ ) by four repetitions of drop jumps. The rest interval between the complex pairs was 4 minutes. The second experimental group (EX2) completed 8 sets of 4 repetitions of half-squat jumps with a load corresponding to a minimum of $90 \%$ of $\mathrm{P}_{\max }$ measured during the pre-test, with 2-3 minutes rest interval between sets. After 8 sets of half-squat jumps, the EX2 subsequently executed 8 sets of 4 repetitions of drop jumps, with a 1-minute rest interval between the sets. Groups EX1 and EX2 conducted an identical total number of repetitions of each exercise.

\section{Subjects}

Thirty male students of the Faculty of Sports Studies were divided into 2 experimental (EX1, $\mathrm{n}=8$, age $=21.4 \pm 1.8$ years, height $=179.3 \pm 5.2 \mathrm{~cm}$, weight $=77.4 \pm 8.4 \mathrm{~kg}$; and EX2, $\mathrm{n}=10$, age $=21.8 \pm 2.6$ years, height $=178.9 \pm 4.0 \mathrm{~cm}$, weight $=75.6 \pm 8.9 \mathrm{~kg})$ and 1 control groups $(\mathrm{CNTR}$, $\mathrm{n}=12$, age $=21.7 \pm 2.2$ years, height $=180.5 \pm 7.0 \mathrm{~cm}$, weight $=75.9 \pm 9.4 \mathrm{~kg}$ ) by the parameter $\mathrm{P}_{\max }$ and also there were no statistical differences between these three groups in the CMJ performance. The original number of 32 people was divided into experimental groups of 10 people, and the 2 remaining people were assigned to the control group rather than to the experimental ones, to prevent potential non-completion of the research. Two people dropped out of the first group (one due to school duties and one due to research unrelated injuries). The control group was asked not to participate in any non-school strength sports activities that they did not have in common with the members of the experimental groups. All participants were informed of the training and testing protocols, the experimental risks, and signed an informed consent form before the research. The consent forms were consistent with the ethical standards and approved by the Ethics Committee of Masaryk University, Brno, Czech Republic.

\section{Measurements}

Two weeks before the intervention started, we informed the subjects about the benefits and risks of this research and the training schedule. There were also training sessions to teach the proper squat technique (as well as the half-squat jump technique), depth jump, and vertical CMJ technique. One week before the training began, the performance in CMJ, maximal power output during loaded half squats and the optimal drop height for drop jumps was tested. The participants completed each test after a minimum of 48 hours of rest. The subjects performed the same warm-up protocol before each training session and testing. The protocol included 5 minutes of low-intensity running and dynamic stretching exercises focused on the whole body ( 3 exercises) and the lower body (5 exercises).

$\mathrm{CMJ}$ testing was conducted before the first training session of the week. They performed two warm-up jumps under the same conditions as in testing (with the rest period of 30-60 seconds). For the test, each subject performed 3 jumps and the best maximum in terms of height was taken for further analysis. The rest period between the jumps was always between 30-60 seconds. Each subject's CMJ height was evaluated using the Myotest accelerometric system (Myotest SA, Sion, Switzerland). The Myotest system is a valid and reliable method to assess vertical jump height (Casartelli, Müller, \& Maffiuletti, 2010; Nuzzo, Anning, \& Scharfenberg, 2011). The CMJ was performed from an upright position with hands kept on the hips for the whole duration of the jump 
to negate the effect of an arm swing. The subjects flexed their knees and hips into a squat position to touch a bench (the height was set individually to $90^{\circ}$ at the knee joint) and then extended their knees and hips into an upward - vertical jump immediately after the touch, without any delay. Each subject was given external encouragement throughout all the tests. The verbal instructions were to jump as high as they could explosively after they had touched the edge of the bench.

To establish the intensity for the loaded half squats (the bottom position at $90^{\circ}$ at the knee joint) a diagnostics series measured by Fitro Dyne Premium (FiTRO Dyne Premium; FiTRONiC Diagnostic and Training Systems Ltd., Bratislava, Slovakia) was used, as it is suitable for measuring muscle power with high reliability (Jennings, Viljoen, Durandt, \& Lambert, 2005). The subjects performed a loaded half-squat jump with a barbell held on the shoulders. The test started with a load of $20 \mathrm{~kg}$ and increased by $10 \mathrm{~kg}$ increments until the average power during the repetition no longer surpassed the previous value. The maximum jump squat power in this study was defined from the highest measured average concentric power. The squat depth before the jump was individually controlled using foam cubes at the bench, where they touched them with their hamstrings and glutes at the $90^{\circ}$ knee angle. The rest interval between each load was 2-3 min. One attempt at each load was given until they no longer outperformed the previous best result in the average concentric power. In the case of not outperforming, they had one more attempt at the same load. When they succeeded, they could continue the testing. If they failed twice with the same weight and did not exceed the previous best result, the testing ended. The average concentric power was determined using a FiTRO Dyne diagnostic device (FiTRO Dyne Premium; FiTRONiC Diagnostic and Training Systems Ltd., Bratislava, Slovakia) attached to the barbell grip to measure displacement. The system's sensor unit is connected to a personal computer and the accompanying software (FiTRO Premium; FiTRONiC Diagnostic and Training Systems Ltd.) gives instantaneous feedback regarding force, velocity, and power (Vanderka, Krčmár, Longová, \& Walker, 2016).

The optimal drop height for depth jumps, the second exercise in the complex pair, was tested before the experiment started. The optimal drop height was identified by testing maximal jump height, which is the recommended method for establishing drop height by Byrne, Moran, Rankin, \& Kinsella (2010).

\section{Statistical Analyses}

The data were analyzed to validate the differences between the pre-test and post-test in the EX1, EX2, and CNTR group through the non-parametric Wilcoxon Signed-Rank Test. To find the differences between weeks and groups the Mann-Whitney U test, Kruskal-Wallis H test (independent group), and Friedman's test (paired group) were used. In addition to statistical significance (W, $\mathrm{U}, \mathrm{H}, \chi^{2}$ ), we also calculated the statistical significance of the effect size (ES) to determine the effect of the differences between two groups (effect size index $d$ ), more independent groups (partial eta-squared, $\eta_{p}^{2}$ ), paired groups (Kendall's $W$ test), and the association between two variables $(r)$. According to Cohen (1988), the effect can be interpreted as small $\left(\eta_{p}^{2}=0.01, d=0.20, \mathrm{r}=0.10, \mathrm{~W}\right.$ $=0.1)$, medium $\left(\eta_{p}^{2}=0.06, d=0.50, \mathrm{r}=0.30, \mathrm{~W}=0.30\right)$, or large $\left(\eta_{p}^{2}=0.14, d=0.80, \mathrm{r}=0.50, \mathrm{~W}\right.$ $=0.50)$. Values less than small were marked as trivial. Using linear regression analysis, we could further predict the height of the CMJ (dependent variable) with a week of training (independent variable). The statistical significance level ( $\alpha$ ) was set to 0.05 . All statistical calculations were performed on IBM SPSS Statistics licensed software (version 25). 


\section{RESULTS}

The main purpose of the study was to compare which training method of complex training leads to better improvements in the performance in the CMJ in 8 weeks. Table 1 contains an overview of the CMJ values divided into three groups (EX1, EX2, CNTR). The results show that there are no statistically significant differences between the groups (EX1, EX2, CNTR) in the pre-test (p $>0.05$ ), but in the post-test, there were statistically significant differences between the CNTR group and the EX2 group $(\mathrm{p}<0.05)$. According to the ES, there was a small effect of the differences between the EX1, EX2, and CNTR group for the pre-test $\left(\eta_{p}^{2}=0.03, \mathrm{CI}=0.00-0.16\right)$ and a large effect of the differences between the EX1, EX2, and control group for the post-test $\left(\eta_{p}^{2}=\right.$ $0.30, \mathrm{CI}=0.03-0.49$ ). To evaluate the differences between the pre-test and post-test in the groups (EX1, EX2, CNTR) we used the Wilcoxon signed-rank test. The results show that EX1 and EX2 lead to a statistically significant improvement after 8 weeks of training $(p>0.05)$ with a small ES. The CNTR group also shows a significant improvement, but according to the trivial effect as a result of the Effect size index $d$ (Cohen's $d$ ) of the difference between pre-test and post-test for a control group, it is classified as an insignificant improvement. Furthermore, Table 1 shows the percentage difference for the differences between the average values of the pre-test and post-test together with statistical (Wilcoxon Singed-Rank Test, Kruskal-Wallis H test) and the effect size (Eta-Square test, Effect size index d, 95\% Confidence Interval) evaluation.

Table $1 \mathrm{CMJ}$ values for experimental and control groups in the pre-test and the post-test

\begin{tabular}{|c|c|c|c|c|c|c|c|}
\hline \multirow{2}{*}{ Groups } & \multirow{2}{*}{$n$} & \multicolumn{2}{|c|}{ Countermovement jump (CMJ) } & \multirow{2}{*}{$\%$ diff } & \multirow{2}{*}{$Z$} & \multirow{2}{*}{$p$} & \multirow{2}{*}{$d(95 \% \mathrm{CI})$} \\
\hline & & Pre-test $(\mathrm{cm})$ & Post-test $(\mathrm{cm})$ & & & & \\
\hline EX1 & 8 & $40.49 \pm 5.04$ & $42.03 \pm 3.41$ & 8.96 & -1.55 & .12 & $0.36(-0.63 ; 1.35)$ \\
\hline $\mathrm{EX} 2$ & 10 & $42.48 \pm 4.00$ & $44.29 \pm 3.80^{\mathrm{b}}$ & 4.17 & -1.90 & .06 & $0.46(-0.42 ; 1.35)$ \\
\hline CNTR & 12 & $39.51 \pm 3.82$ & $39.18 \pm 2.49^{\mathrm{a}}$ & 0.84 & -0.65 & .51 & $0.10(-0.70 ; 0.90)$ \\
\hline $\mathrm{H}$ & & 2.68 & 10.00 & & & & \\
\hline $\mathrm{p}$ & & .26 & .01 & & & & \\
\hline$\eta_{p}^{2}(95 \% \mathrm{CI})$ & & $0.03(0.00 ; 0.16)$ & $0.30(0.03 ; 0.49)$ & & & & \\
\hline
\end{tabular}

Note: $Z=\mathrm{z}$-score for Wilcoxon Signed-Rank Test, $p=\mathrm{p}$-value, $d=$ effect size index $d$ (Cohen's $d$ ), CI $=$ confidence interval, $\mathrm{H}=$ Kruskal-Wallis $\mathrm{H}$ test, $\eta_{p}^{2}=$ partial eta-square, ${ }^{\mathrm{a}}=$ significant difference with EX2, ${ }^{\mathrm{b}}=$ significant difference with CNTR

The second purpose of this study_was to compare the changes in performance measured every week before the first training session of the week. In Figure 1, the differences between the groups are recorded. The changes have a wave-like character. In Figure 1 we can observe that after the first week of the intervention the muscle demands on the probands were very high, therefore the performance in the second week is lower than in the pre-test. Subsequently, the load on the probands was increased by continuous testing of the ability to train the half-squat jumps, always above $90 \%$ of the current Pmax. However, in the 3rd and 4th weeks, after the first neuromuscular adaptation, the increase was very high and lead to high muscle damage. That is probably the reason for the fluctuation in performance for the CMJ in the 4th and 5th week. The same situation was repeated after week 7. It can be seen graphically in the 9th week when the post-testing was done during and at the end of the week, the probands were able to perform at their best. 


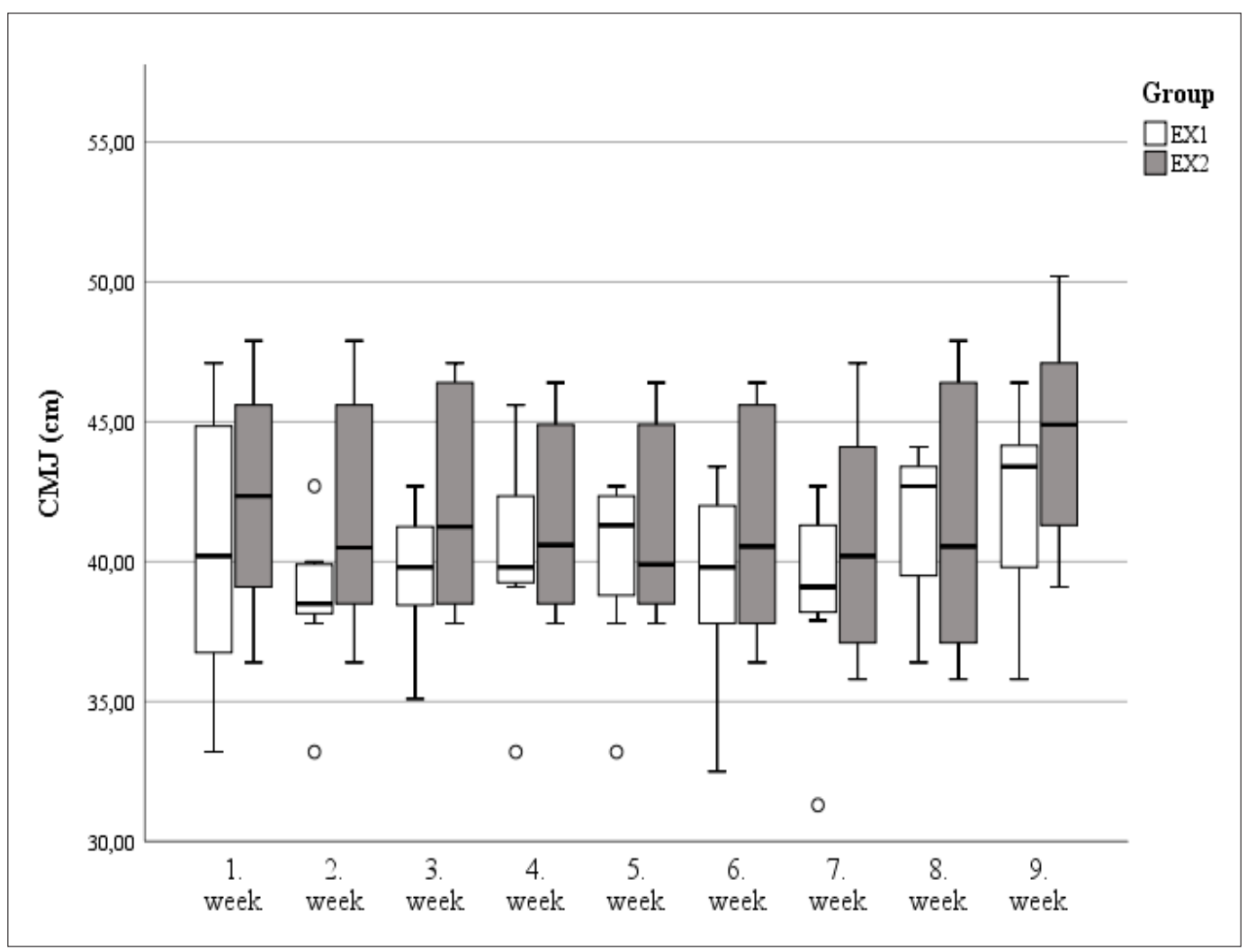

Figure 1 Overview of CMJ performance changes in experimental groups

Table 2 shows the differences between the experimental groups EX1 and EX2 each week. The hypothesis was tested by Friedman's test, Mann-Whitney U test, and Effect size by Kendall's W test, Cohen's $d, 95 \%$ confidence interval. We reject the null hypothesis for the difference between the values for the CMJ by week according to EX1 and EX2 training program, because there was a statistically significant difference between the value of CMJ $(\mathrm{cm})$ each week for $\operatorname{EX1}\left(\chi^{2}(8)=\right.$ $17.9, p=0.02, \mathrm{ES}=$ small effect; $\mathrm{EX} 2\left(\chi^{2}(8)=21.2, p=0.01, \mathrm{ES}=\right.$ small effect $)$. According to the Mann-Whitney $U$ test we also cannot reject the null hypothesis, because the CMJ $(\mathrm{cm})$ wasn't greater for EX1 $\left(\mathrm{Med}_{\max }=43.05, \mathrm{Med}_{\min }=39.15\right)$ than for EX2 $\left(\mathrm{Med}_{\max }=44.90, \mathrm{Med}_{\text {min }}=39.90\right)$, $\mathrm{U}_{1-9 \text { week }}=39.5-53.5, p>0.05$. The results show that there are significant differences in performance in the CMJ over the weeks between EX1 and EX2 $(\mathrm{p}<0.05)$. But there were no significant differences between the experimental groups (EX1, EX2). 
Table 2 Differences between the values of CMJ by week according to EX1 and EX2 training program

\begin{tabular}{cccccc}
\hline \multirow{2}{*}{ No. of week } & \multicolumn{2}{c}{ Experimental group } & \multirow{2}{*}{$U$} & $p$ & $d(95 \% \mathrm{CI})$ \\
\cline { 2 - 3 } & EX1 & EX2 & & & \\
\hline 1. & $40.5(5.0)$ & $42.5(4.0)$ & 50.00 & .41 & $0.5(-0.5 ; 1.4)$ \\
2. & $39.5(3.6)$ & $41.6(4.2)$ & 51.5 & .31 & $0.5(-0.4 ; 1.5)$ \\
3. & $40.1(2.8)$ & $42.1(3.8)$ & 50.0 & .41 & $0.6(-0.3 ; 1.6)$ \\
4. & $40.5(3.6)$ & $41.6(3.6)$ & 41.5 & .90 & $0.3(-0.6 ; 1.3)$ \\
5. & $40.4(3.4)$ & $41.4(3.7)$ & 44.5 & .70 & $0.3(-0.6 ; 1.2)$ \\
6. & $39.5(3.6)$ & $41.2(3.9)$ & 47.0 & .57 & $0.5(-0.5 ; 1.4)$ \\
7. & $39.0(8.0)$ & $40.8(4.0)$ & 47.5 & .52 & $0.3(-0.6 ; 1.2)$ \\
8. & $41.3(2.7)$ & $41.5(4.7)$ & 39.0 & .97 & $0.1(-0.8 ; 1.0)$ \\
9. & $42.0(3.4)$ & $44.3(3.8)$ & 53.5 & .24 & $0.7(-0.3 ; 1.6)$ \\
$n$ & 8 & 10 & & & \\
$\chi^{2}$ & 17.9 & 21.2 & & & \\
$p$ & .02 & .01 & & & \\
W & 0.28 & 0.27 & & & \\
\hline
\end{tabular}

Note: $U=$ Mann-Whitney $\mathrm{U}$ test, $p=$ p-value, $d=$ effect size index $d$ (Cohen's $d), \mathrm{CI}=$ confidence interval, $\chi^{2}=$ Friedman's test, $\mathrm{W}=$ Kendall's $\mathrm{W}$ test

The third purpose of this study was to find out what prediction model results from the performances in the CMJ of EX1 and EX2 which varied from week to week. The regression analyses (Table 3 ) show that there is a small association $\left(r=0.104, r^{2}=1.08 \%\right)$ between the performance in the $\mathrm{CMJ}$ and the week of the training program in EX1, and a trivial $\left(\mathrm{r}=0.043, \mathrm{r}^{2}=0.18 \%\right)$ association between the performance in the $\mathrm{CMJ}$ and the week of the training program in EX2. We could consider, that in the EX1 the CMJ performance is increased by $0.14 \mathrm{~cm}(\mathrm{CI}=-0.18-0.46)$ each week, and for EX2 by $0.07 \mathrm{~cm}(\mathrm{CI}=-0.25-0.38)$ each week. However, both models are statistically insignificant $(\mathrm{EX} 1: \mathrm{t}=0.87, \mathrm{p}=0.39$; $\mathrm{EX} 2 \mathrm{t}=0.41, \mathrm{p}=0.69)$. It is important to note that none of the simple linear regression models was statistically insignificant ( $p>05)$.

Table 3 Results of regression analyses between experimental groups (EX1, EX2) and number of weeks

\begin{tabular}{|c|c|c|c|c|c|c|c|c|}
\hline & \multirow{2}{*}{ Model } & \multicolumn{2}{|c|}{$\begin{array}{c}\text { Unstandardized } \\
\text { Coefficients }\end{array}$} & \multirow{2}{*}{$\begin{array}{c}\text { Standardized } \\
\text { Coefficients } \\
\text { Beta }\end{array}$} & \multirow{2}{*}{$t$} & \multirow{2}{*}{$p$} & \multicolumn{2}{|c|}{$95 \%$ CI for B } \\
\hline & & B & Std. Error & & & & $\begin{array}{l}\text { Lower } \\
\text { Bound }\end{array}$ & $\begin{array}{l}\text { Upper } \\
\text { Bound }\end{array}$ \\
\hline \multirow[t]{2}{*}{1} & (Constant_EX1) & 39.60 & 0.90 & & 44.03 & .00 & 37.81 & 41.39 \\
\hline & week & 0.14 & 0.16 & 0.104 & 0.87 & .39 & -0.18 & 0.46 \\
\hline \multirow[t]{2}{*}{2} & (Constant_EX2) & 41.58 & 0.90 & & 46.09 & .00 & 39.78 & 43.37 \\
\hline & week & 0.07 & 0.16 & 0.043 & 0.41 & .69 & -0.25 & 0.38 \\
\hline
\end{tabular}

Note: dependent variable: CMJ (cm, continuous), independent variable: No. of the week (1-9, discrete) 


\section{DISCUSSION}

The study aimed to compare the effects of two different methods of the complex training application. The current recommendations for implementing complex training are focused on the intracomplex rest interval (ICRI), which is the time between the heavy resisted and the light resisted exercises (Jensen \& Ebben, 2003). In acute effect studies, however, minimal rest such as 10 and 15 seconds seems to decrease a subsequent power output (Bevan, Owen, Cunningham, Kingsley, \& Kilduff, 2009), but there is no mention of the effect on chronic adaptation. The examined range of time between the complex pair exercises is from 10 seconds to 24 minutes (Jensen \& Ebben, 2003), and the practical advice for coaches is to apply 2 minutes of the ICRI if focusing on strength, and 1 minute of the ICRI if focusing on power (Carter \& Greenwood, 2014). In our study, we tried to verify whether the low ICRI values in the regular complex training method using complex pairs lead to a better adaptation or stimulation of the body to produce more power output. Contrary to expectations, we did not find any significant increases after 8 weeks of intervention. In any case, it should be noted that the results of the comparison of the groups after the intervention indicate significant differences $(\mathrm{p}<0.05)$ between the control group and the EX2 group (training complex pairs separately). Interestingly, the studies do not describe in detail the effects of training, which is designed as training performed by EX2, where subjects perform several sets of the first exercise from the complex pair and then several sets of the second exercise from the complex pair in one training unit. Studies (Ebben, Jensen, \& Blackard, 2000; Stasinaki, Gloumis, Spengos, Blazevich, Zaras, Georgiadis, ... \& Terzis, 2015) describing the complex training method state that heavy resistance exercises and plyometric training are performed within one training unit, but this almost always means utilizing complex pairs (heavy resistance exercise first, then plyometric exercise with adequate ICRI using PAP).

If we compare the usefulness of complex training methods in training, we must also take into account the possible different lengths of training with different methods of implementing complex training. The research of Santos \& Janeira (2008) states time efficiency as one of the main benefits of this method. In our study, we compared the time-effectiveness of a method using complex pairs (EX1) and a mixed concept of a training unit, which includes both complex pair exercises done separately to increase muscle strength and power, and plyometric exercises. Therefore, we calculated rest intervals during training and compared them between the groups, assuming that the time required to perform the same exercise was the same. EX1 had 8 sets (rest interval after the set was 4 minutes), each consisting of 4 half-squat jumps and 4 depth jumps with a rest interval of up to 15 seconds. The total time of the rest intervals is 23 minutes in the EX1 group. EX2 had 8 sets of 4 half-squat jumps with a rest interval of 2-3 min after each set, and then 8 sets of 4 depth jumps with a rest interval of 1 minute after each set. The total time of rest intervals is from 23 to 31 minutes depending on the rest interval between the half-squat jumps in the EX2 group. Thus, the complex pair method saves from 0 to 8 minutes. When using multiple complex pairs during the training, the time saved should be considerable and useful.

Another possible view of the results is the use of suitable statistical methods and interpretation of the results. For athletes who are experienced in strength or explosive training, we cannot expect performance gains as high as we would expect from untrained individuals. For elite athletes, even very small improvements play a role, as long as there are no big differences in the results between the top athletes of the sport. Thus, the observed, although statistically insignificant ( $\mathrm{p}>$ $0.05)$, average performance increases in CMJ over 8 weeks were $0.14 \mathrm{~cm}(\mathrm{CI}=-0.18-0.46)$ each week for EX1, and $0.07 \mathrm{~cm}(\mathrm{CI}=-0.25-0.38)$ each week for EX2, which could play a key role at the elite level. 
This study had several limitations that must be considered when extrapolating conclusions based on the results. First, the participants were divided into the groups by the parameter of $P_{\text {max }}$ and not primarily by their CMJ performance, although the CMJ performance in the pre-test did not differ statistically significantly among all groups. Second, the participants had experience with resistance training, but we did not take into account the differences in years of experience, experience with different forms of training, or their history of training and skill levels for half-squat and depth jumps. Third, an effort to ensure that the training intensity was maintained above $90 \% \mathrm{P}_{\text {max }}$ could lead to some individuals being able to train above and below the level in the last series of squat-jump exercises. These high intensities may be the cause of the unconvincing results at the end of this study when in the last two weeks we probably no longer had to regulate the intensity of the squat-jump load. Fourth, a heavy resistance exercise to elicit PAP is not generally defined as exceeding $90 \%$ of $\mathrm{P}_{\max }$.

\section{CONCLUSION}

Due to the limited number of participants and ambiguous results, we cannot generalize the results of the study. However, the results suggest that when using complex pairs, it is necessary to consider and use a longer time interval between the exercises in a complex pair (more than 15 seconds). The analysis indicated the possible effectiveness of the training of the EX2 group (separate pairs of complex training), which differed statistically significantly from the control group in the post-test $(\mathrm{p}<0.05)$. Subsequently, we wanted to create a prediction model, but we failed because our results were not statistically significant. Our practice recommendations are that when using complex pairs, the trainer can save training time and increase training intensity, and the training will be similarly effective as when complex training is applied in the form of separate exercises of complex pairs in the same training session. However, an additional longer-term intervention experiment is needed to determine the effect of performing exercises of complex pairs separately in one training session.

\section{Acknowledgments}

The implementation of the research was approved by the Ethics Committee for Research of Masaryk University in Brno.

\section{References}

Bevan, H. R., Owen, N. J., Cunningham, D. J., Kingsley, M. I., \& Kilduff, L. P. (2009). Complex training in professional rugby players: Influence of recovery time on upper-body power output. The Journal of Strength \& Conditioning Research, 23(6), 1780-1785.

Bompa, T. (1983). Theory and methodology of training Duduque. lowa. Kendall/Hunt.

Byrne, P. J., Moran, K., Rankin, P., \& Kinsella, S. (2010). A comparison of methods used to identify 'optimal'drop height for early phase adaptations in depth jump training. The Journal of Strength \& Conditioning Research, 24(8), 2050-2055.

Carter, J., \& Greenwood, M. (2014). Complex training reexamined: Review and recommendations to improve strength and power. Strength \& Conditioning Journal, 36(2), 11-19.

Casartelli, N., Müller, R., \& Maffiuletti, N. A. (2010). Validity and reliability of the Myotest accelerometric system for the assessment of vertical jump height. The Journal of Strength \& Conditioning Research, 24(11), 3186-3193. doi: 10.1519/ JSC.0b013e3181d8595c

Chu, D. A. (1998). Jumping into plyometrics. Human Kinetics.

Cohen, J. (1988). Statistical power analysis for the behavioural sciences (2. Ed.). Hillsdale, NJ: Erlbaum.

Docherty, D., Robbins, D., \& Hodgson, M. (2004). Complex training revisited: A review of its current status as a viable training approach. Strength and Conditioning Journal, 26(6), 52-57. 
Ebben, W. P. (2002). Complex training: A brief review. Journal of sports science \& medicine, 1(2), 42-46.

Ebben, W. P., Jensen, R. L., \& Blackard, D. O. (2000). Electromyographic and kinetic analysis of complex training variables. The Journal of Strength \& Conditioning Research, 14(4), 451-456.

Faigenbaum, A. D., Kraemer, W. J., Blimkie, C. J., Jeffreys, I., Micheli, L. J., Nitka, M., \& Rowland, T. W. (2009). Youth resistance training: updated position statement paper from the national strength and conditioning association. The Journal of Strength \& Conditioning Research, 23, S60-S79.

Hodgson, M., Docherty, D., \& Robbins, D. (2005). Post-activation potentiation. Sports medicine, 35(7), 585-595.

Jennings, C. L., Viljoen, W., Durandt, J., \& Lambert, M. I. (2005). The reliability of the FitroDyne as a measure of muscle power. Journal of Strength and Conditioning Research, 19(4), 859-863.

Jensen, R. L., \& Ebben, W. P. (2003). Kinetic analysis of complex training rest interval effect on vertical jump performance. Journal of Strength and Conditioning Research, 17(2), 345-349.

Molina-Garcia, P., Plaza-Florido, A., Mora-Gonzalez, J., Torres-Lopez, L. V., Vanrenterghem, J., \& Ortega, F. B. (2020). Role of physical fitness and functional movement in the body posture of children with overweight/obesity. Gait \& Posture (pre-proof article). https://doi.org/10.1016/j.gaitpost.2020.04.001

Niemann, M. J., Tucker, L. A., Bailey, B. W., \& Davidson, L. E. (2020). Strength Training and Insulin Resistance: The Mediating Role of Body Composition. Journal of Diabetes Research, 2020. https://doi.org/10.1155/2020/7694825

Nuzzo, J. L., Anning, J. H., \& Scharfenberg, J. M. (2011). The reliability of three devices used for measuring vertical jump height. The Journal of Strength \& Conditioning Research, 25(9), 2580-2590. doi: 10.1519/JSC.0b013e3181fee650

Ozen, G., Atar, O., \& Koc, H. (2020). The Effects of A 6-Week Plyometric Training Programme on Sand Versus Wooden Parquet Surfaces on the Physical Performance Parameters of Well-Trained Young Basketball Players. Montenegrin Journal of Sports Science and Medicine, 9(1), 27-32. doi: 10.26773/mjssm.200304

Robbins, D. W. (2005). Postactivation potentiation and its practical applicability: a brief review. Journal of Strength and Conditioning Research, 19(2), 453-458. doi:10.1519/R-14653.1

Rowland, T. W. (2007). Promoting physical activity for children's health. Sports Medicine, 37(11), 929-936.

Sale, D. G. (2002). Postactivation potentiation: role in human performance. Exercise and sport sciences reviews, 30(3), 138-143.

Santos, E. J., \& Janeira, M. A. (2008). Effects of complex training on explosive strength in adolescent male basketball players. The Journal of Strength \& Conditioning Research, 22(3), 903-909.

Stasinaki, A. N., Gloumis, G., Spengos, K., Blazevich, A. J., Zaras, N., Georgiadis, G., ... \& Terzis, G. (2015). Muscle strength, power, and morphologic adaptations after 6 weeks of compound vs. complex training in healthy men. The Journal of Strength \& Conditioning Research, 29(9), 2559-2569.

Vanderka, M., Krčmár, M., Longová, K., \& Walker, S. (2016). Acute effects of loaded half-squat jumps on sprint running speed in track and field athletes and soccer players. Journal of strength and conditioning research, 30(6), 1540-1546. doi: $10.1519 /$ JSC.0000000000001259

Zeng, J., Peng, L., Zhao, Q., \& Chen, Q. G. (2020). Effects over 12 weeks of different types and durations of exercise intervention on body composition of young women with obesity. Science \& Sports. doi: 10.1016/j.scispo.2019.10.011

\section{Contact Information}

Mgr. Tereza Králová, email: tereza.kralova@fsps.muni.cz; Department of Athletics, Swimming and Outdoor Sports. 